\title{
Estudo comparativo do nível de qualidade de vida entre sujeitos acondroplásicos e não-acondroplásicos
}

\section{Comparative study of quality of life level between achondroplasics and non-achondroplasics subjects}

Mariana Pereira Cervan', Márcia Cristina Pires da Silva', Rodrigo Lopes de Oliveira Lima', Roberto Fernandes da Costa'

\section{RESUMO}

A acondroplasia é caracterizada como um distúrbio genético autossômico dominante que afeta a ossificação endocondral, constituindo uma das causas de nanismo. Entre as características está presente principalmente a baixa estatura e desproporção tronco/membros. Diante disso, o acondroplásico poderá se mostrar inferiorizado e insatisfeito com sua aparência física, influenciando, juntamente a outros fatores, na qualidade de vida (QV) dessa população. Objetivo: Comparar o nível de QV entre sujeitos acondroplásicos e não-acondroplásicos. Método: Foram avaliados 21 indivíduos acondroplásicos, sendo nove homens e 13 mulheres, pareados por idade e gênero, a 21 não-acondroplásicos. O nível de QV foi estimado por meio do questionário WHOQOL-BREF. Resultados: Na comparação entre sujeitos acondroplásicos e não acondroplásicos do gênero feminino constatou-se que no domínio físico as mulheres acondroplásicas apresentaram escore médio significativamente menor, enquanto na comparação entre os gêneros, o grupo de mulheres acondroplásicas apresentou escore médio significativamente menor que os homens acondroplásicos no domínio psicológico. Conclusão: No presente estudo, a QV não apresentou diferença entre os grupos, entretanto, nos domínios físico e psicológico, as mulheres acondroplásicas demonstraram menor satisfação com sua condição.

\begin{abstract}
Achondroplasia is characterized as an autosomal dominant genetic disturbance which affects the endochondral ossification which is a common cause of dwarfism syndrome. Among the characteristics the most common are the short stature and disproportional trunk/limbs. Thus, the achondroplasic subjects can face themselves inferior and not satisfied with their physical appearance, influencing, among other factors, the quality of life of this population. Objective:

To compare the quality of life level between achondroplasic and non-achondroplasic subjects.

Methods: Study with 21 achondroplasics in which, nine were men and 13 women, paired by age and gender to 21 non-achondroplasic subjects. The quality of life level was estimated by
\end{abstract}

\section{Palavras-chaves}

Acondroplasia, qualidade de vida, WHOQOL-BREF. 


\section{Keywords}

Achondroplasia, quality of life, WHOQOL-BREF.
WHOQOL-BREF questionnaire. Results: In the comparison between achondroplasic and nonachondroplasic females we verified that in physical domain achondroplasic women presented mean score significantly lower than non-achondroplasic. In the comparison between genders, achondroplasic females presented mean score significantly lower than achondroplasic males in psychological domain. Conclusion: In this study, the quality of life didn't present any difference between groups, however, in physical and psychological domains, achondroplasic women demonstrated less satisfaction to their condition.

\section{INTRODUÇÃO}

A acondroplasia é a mais freqüente displasia esquelética de membros curtos, resultante de uma mutação genética que afeta a ossificação endocondral. O processo básico é uma inabilidade da placa epifisária em produzir cartilagem colunar, o que resulta um crescimento insuficiente dos ossos longos, tornando a linha de ossificação irregular e alterando o fechamento das epífises'.

Tal displasia é caracterizada como um distúrbio autossômico dominante, no qual cerca de $80 \%$ a $90 \%$ dos casos são representados por novas mutações, o que implica que majoritariamente os pais de filhos acondroplásicos não apresentam a mutação gênica. Apesar de os progressos do Projeto Genoma Humano, no início da década de 1990, terem propiciado mais conhecimentos a respeito da caracterização genética na acondroplasia, foi somente em 1994 que foi localizada a posição exata da mutação gênica no braço curto do cromossomo quatro ${ }^{2}$. Segundo Rousseau et al. ${ }^{3}$, a mutação do gene foi localizada no receptor do fator de crescimento do fibroblasto tipo 3 (FGFR3), no qual a maioria dos pacientes com sintomatologia clínica de acondroplasia típica apresentou a mesma mutação. Ocorre substituição de uma arginina por uma glicina no domínio transmembrana do receptor, que está situado nos condrócitos na placa de crescimento dos ossos.

Geralmente, o genótipo dos acometidos é heterozigótico, pois, segundo Griffiths et al. ${ }^{4}$, acredita-se que a homozigose seja bastante grave e até mesmo letal. Assim, são conhecidos poucos casos homozigóticos gravemente afetados, que são resultantes da união de heterozigotos e que foram a óbito precocemente ${ }^{5}$.

A incidência foi estimada nos Estados Unidos em 15 casos para cada um milhão de nascidos vivos, contudo alguns autores afirmam que outras condições semelhantes à acondroplasia possam ter influenciado no referido cálculo. Estima-se que a incidência deva estar entre um caso para cada 8.000 a 10.000 nascidos vivos ${ }^{6,7}$

A partir dos anos 1970, a atenção sobre esta patologia centrou-se em estudar as complicações que aparecem ao longo do tempo. A acondroplasia é bastante uniforme quanto à expressão clínica, mas podem haver variações em relação à gravidade de cada deformidade ${ }^{8}$. Quando transmitida por herança, as mesmas características clínicas do progenitor tendem a se fazer presentes no filho?.

O fenotipo dos acondroplásicos se caracteriza principalmente pela baixa estatura, desproporção tronco/membros, com membros curtos e predomínio do segmento proximal, calota craniana normal, fisionomia característica com fronte proeminente e depressão da ponte nasal. A mandíbula é ressaltada e grande em relação aos ossos da face, bem como os dentes são sobrepostos e mal alinhados gerando má oclusão ${ }^{10}$.

As mãos são pequenas e largas, e os dedos curtos com separação entre a terceira e quarta falanges (mão em tridente) $)^{11}$.

A postura do paciente se caracteriza pela acentuação da cifose torácica e da lordose lombar, com abdome protuso, e o surgimento de hérnia umbilical não é raro. Alguns autores ainda citam o surgimento de uma cifose toracolombar, ${ }^{8,12}$.

Não há prejuízo do desenvolvimento mental, mesmo nos casos que apresentam a referida hidrocefalia, que pode estar presente na infância. A inteligência não é afetada e muitos acondroplásicos são, na verdade, dotados de inteligência acima da média13,14.

São descritas ainda inúmeras complicações neurológicas, como estenose do forame magno; respiratórias (apnéia do sono, obstrução de via aérea superior, cor pulmonale etc.) $)^{15}$; ergonômicas e sociais ${ }^{16}$.

Embora a acondroplasia seja considerada uma doença associada à longevidade normal e, portanto, compatível com uma vida íntegra e produtiva, muitos autores têm documentado que essas várias complicações clínicas e sociais podem comprometer esse panorama. Diante disso, eventualmente, muitos acondroplásicos poderão apresentar distúrbios psicológicos decorrentes do seu complexo de inferioridade e insatisfação com a aparência física ${ }^{17}$.

Em um estudo realizado por Mahomed et al. ${ }^{16}$, foi relatado que adultos acondroplásicos na etária dos 16 aos 54 anos, apresentaram excelente estado geral mental, e meIhor, se comparado ao grupo-controle.

Folstein et al. ${ }^{18}$ atribuem tais resultados ao fato de que pessoas que já nascem com um distúrbio evidente possam vir a ser menos críticos em relação às suas limitações, se 
comparados àqueles que adquiriram uma doença durante a adolescência ou na fase adulta. Além disso, muitos desses pacientes estão envolvidos desde cedo em dinâmicas de grupo, o que possivelmente contribui com a auto-estima e com a adaptação de suas habilidades.

A participação religiosa tem significado importante para a aceitação das diferenças, sendo relatado que indivíduos com doenças físicas crônicas utilizam a religião para enfrentar positivamente suas situações ${ }^{19}$. A prática religiosa tende a tornar os indivíduos mais saudáveis e, conseqüentemente, a estender a expectativa de vida de acordo com Koenig20; assim, estudo realizado com indivíduos acondroplásicos praticantes religiosos mostrou que eles apresentaram, com maior freqüência, melhor percepção de suas condições, visto que a religião promoveu maior envolvimento com a comunidade, facilitando a aceitação perante as diferenças ${ }^{21}$.

De acordo com Holmes et al. ${ }^{22}$, no início da puberdade há uma tendência de as atividades sociais se reduzirem e os amigos se afastarem; porém, os adolescentes acondroplásicos apresentaram melhores resultados nos parâmetros de depressão e vitalidade em relação aos seus pares não acometidos.

Hoje em dia quem não se enquadra em um padrão convencional de beleza, peso ou estatura, faz parte de um grupo estigmatizado da sociedade; e os anões por alcançam a estatura entre $70 \mathrm{~cm}$ e $140 \mathrm{~cm}$ no máximo, na fase adulta, fazem parte desse grupo. Este fator associado à falta de acessibilidade em serviços, ambientes e transportes públicos, contribui para a exclusão social e a manutenção do preconceito. Essas atitudes discriminatórias são indícios de desconhecimento pejorativo diante das diferenças, no qual muitas vezes são faltados, aos anões, o respeito e o reconhecimento como pessoa, sendo tratados de forma infantilizada e muitas vezes ridicularizados.

De acordo com Gollust et al. ${ }^{21}$, as principais desvantagens citadas pelos acondroplásicos não são suas limitações físicas, mas sim a percepção da sociedade frente às suas condições. Muitos relataram provocações, grosserias, discriminações, entre outras formas de preconceito, como sendo as piores dificuldades encontradas para o envolvimento com a sociedade, o que prejudica o convívio social.

Com isso, tais fatores podem influenciar na QV dessa população, que inclui uma variedade de condições que podem afetar a percepção desse indivíduo, suas relações interpessoais e o ambiente em que ele vive, incluindo, mas não se limitando apenas à sua condição de saúde.

Neste sentido, o objetivo do presente estudo foi analisar os níveis de QV de sujeitos acondroplásicos e compará-los aos de não-acondroplásicos de mesma idade e gênero.

\section{MÉTODO}

\section{Casuística}

O estudo contou com a participação de 21 indivíduos acondroplásicos da Associação Gente Pequena do Brasil (AGPB), sendo nove homens e 13 mulheres, em uma faixa etária dos 14 aos 53 anos, pareados por idade e gênero a 21 indivíduos não-acondroplásicos.

\section{Procedimentos e instrumentos}

O nível de QV foi estimado por meio do questionário WHOQOL-BREF, proposto pela Organização Mundial de Saúde (OMS) em 1995, cuja versão em português foi validada por Fleck et al.23. Esse instrumento de 26 questões analisa os domínios físico, psicológico, social e ambiental separadamente, além de propor um resultado total, atribuindo valores a cada um que variam de 50 a 100. O domínio físico se refere às possíveis dores e desconfortos, fadiga, sono e repouso; no domínio psicológico, avalia-se a auto-estima, a imagem corporal e aparência, os sentimentos positivos e negativos, assim como a capacidade para aprender e se concentrar; o domínio social refere-se ao nível de independência considerando-se a mobilidade, a capacidade para o trabalho, bem como a dependência de medicação ou de tratamentos do indivíduo; e, finalmente, no domínio ambiental são analisadas as relações pessoais, a atividade sexual, os recursos financeiros, a segurança física e proteção, os transportes, as oportunidades de lazer, a religião etc.

As questões do WHOQOL-BREF foram formuladas para uma escala de respostas do tipo Likert, com uma escala para intensidade (nada - extremamente), capacidade (nada - completamente), freqüência (nunca - sempre) e avaliação (muito insatisfeito - muito satisfeito / muito ruim - muito bom).

Aqueles que aceitaram participar voluntariamente do estudo assinaram um termo de consentimento livre e esclarecido e foram devidamente orientados que durante o preenchimento do instrumento não deveriam estar acompanhados de outras pessoas, seja cônjuge, familiares, amigos ou até mesmo do entrevistador para que não ocorressem possíveis influências sobre as respostas. Além disso, foi enfatizado que o questionário se referia às duas últimas semanas, independentemente do local onde o indivíduo se encontrasse.

O estudo obedeceu aos princípios éticos para pesquisa envolvendo seres humanos, conforme a resolução 196/96 do Conselho Nacional de Saúde e foi aprovado pelo Comitê de Ética em Pesquisa da Universidade Santa Cecília.

\section{Tratamento estatístico}

O pressuposto de distribuição normal dos dados foi verificado pelo teste de Kolmogorov-Smirnov. Considerando que todas as variáveis apresentaram distribuição normal, foi utilizado o teste t de Student para comparar os resultados médios dos domínios, tanto entre os grupos quanto entre os gêneros, sendo que foi adotada significância estatística para $\mathrm{p}<0,05$. 


\section{RESULTADOS}

As características dos grupos avaliados são apresentadas nas tabelas 1 e 2.

Os resultados obtidos no WHOQOL-BREF são apresentados nas tabelas 3 a 6 .

Tabela 1. Características dos indivíduos acondroplásicos de ambos os gêneros.

\begin{tabular}{lccccccc}
\hline & \multicolumn{3}{c}{ Masculino (9) } & & \multicolumn{3}{c}{ Feminino (13) } \\
\cline { 2 - 3 } \cline { 7 - 8 } & Mínimo & Máximo & Média \pm DP & & Mínimo & Máximo & Média \pm DP \\
\hline Idade & 21,5 & 53,5 & $33,0 \pm 12,01$ & & 14,9 & 51,4 & $32,5 \pm 10,60$ \\
Massa & 32,6 & 63,9 & $48,9 \pm 11,35$ & & 32,2 & 79,0 & $52,3 \pm 12,58$ \\
Estatura & 107,0 & 138,2 & $124,5 \pm 12,79$ & & 113,3 & 130,0 & $122,8 \pm 4,95$ \\
IMC & 26,6 & 36,4 & $31,2 \pm 3,22$ & & 23,7 & 53,1 & $34,8 \pm 8,60$ \\
\hline
\end{tabular}

Tabela 3. Comparação entre acondroplásicos e não-acondroplásicos do gênero masculino para os resultados médios obtidos no WHOQOL-BREF.

\begin{tabular}{lccc}
\hline & Acondroplásicos & Não-acondroplásicos & $\mathbf{p}$ \\
\hline Físico & $76,2 \pm 13,4$ & $78,6 \pm 8,6$ & 0,657 \\
Psicológico & $88,4 \pm 10,2$ & $81,5 \pm 16,7$ & 0,302 \\
Social & $84,3 \pm 6,5$ & $80,6 \pm 11,8$ & 0,421 \\
Ambiental & $67,0 \pm 9,9$ & $66,7 \pm 15,0$ & 0,954 \\
Total & $77,2 \pm 6,4$ & $76,0 \pm 10,6$ & 0,761 \\
\hline
\end{tabular}

Tabela 2. Características dos indivíduos não-acondroplásicos de ambos os gêneros.

\begin{tabular}{lccccccc}
\hline & \multicolumn{4}{c}{ Masculino (9) } & & \multicolumn{3}{c}{ Feminino (13) } \\
\cline { 2 - 3 } \cline { 7 - 8 } & Mínimo & Máximo & Média \pm DP & & Mínimo & Máximo & Média \pm DP \\
\hline Idade & 21,8 & 53,9 & $33,1 \pm 12,20$ & & 14,8 & 50,3 & $32,1 \pm 10,30$ \\
Massa & 75,0 & 105,0 & $87,1 \pm 11,00$ & & 51,0 & 84,0 & $61,9 \pm 9,58$ \\
Estatura & 167,0 & 187,0 & $177,1 \pm 7,10$ & & 155,1 & 170,9 & $162,6 \pm 3,93$ \\
IMC & 21,4 & 35,5 & $27,9 \pm 3,92$ & & 19,7 & 32,0 & $23,5 \pm 3,90$ \\
\hline
\end{tabular}

Tabela 5. Comparação entre os gêneros para os resultados médios obtidos no WHOQOL-BREF para indivíduos acondroplásicos.

\begin{tabular}{lccc}
\hline & Masculino & Feminino & $\mathbf{p}$ \\
\hline Físico & $76,2 \pm 13,4$ & $72,0 \pm 8,5$ & 0,377 \\
Psicológico & $88,4 \pm 10,2$ & $73,7 \pm 14,1$ & 0,010 \\
Social & $84,3 \pm 6,5$ & $73,7 \pm 22,8$ & 0,136 \\
Ambiental & $67,0 \pm 9,9$ & $61,3 \pm 15,3$ & 0,301 \\
Total & $77,2 \pm 6,4$ & $69,6 \pm 11,3$ & 0,058 \\
\hline
\end{tabular}

Tabela 6. Comparação entre os gêneros para os resultados médios obtidos no WHOQOL-BREF para indivíduos não-acondroplásicos.

\begin{tabular}{lccc}
\hline & Masculino & Feminino & $\boldsymbol{p}$ \\
\hline Físico & $78,6 \pm 8,6$ & $81,9 \pm 6,1$ & 0.303 \\
Psicológico & $81,5 \pm 16,7$ & $79,8 \pm 11,6$ & 0,784 \\
Social & $80,6 \pm 11,8$ & $78,2 \pm 16,2$ & 0,714 \\
Ambiental & $66,7 \pm 15,0$ & $70,4 \pm 12,7$ & 0,533 \\
Total & $76,0 \pm 10,6$ & $76,8 \pm 8,3$ & 0,842 \\
\hline
\end{tabular}

Na comparação entre indivíduos acondroplásicos e nãoacondroplásicos do gênero masculino, não houve diferença estatisticamente significante em quaisquer dos domínios, o que possivelmente sugere que tal displasia não interfira na percepção que esse grupo tem de sua QV. Já na comparação entre indivíduos acondroplásicos e não-acondroplásicos do gênero feminino, pôde-se constatar que no domínio físico as mulheres acondroplásicas apresentaram resultados estatisticamente menores $(72,0 \pm 8,5)$ em relação aos resultados obtidos no grupo de mulheres não-acondroplásicas $(81,9 \pm 6,1)$.

A tabela 5 se refere à comparação entre os gêneros para o grupo de acondroplásicos, no qual foi observada diferença estatisticamente significante no domínio psicológico para o gênero feminino $(p=0,010)$. E, finalmente, na comparação entre os gêneros para o grupo de não-acondroplásicos não houve diferença estatisticamente significativa em quaisquer dos domínios da QV, o que possivelmente indica que esta displasia influencia na percepção dos indivíduos acometidos.

\section{DISCUSSÃO}

Tabela 4. Comparação entre acondroplásicos e não-acondroplásicos do gênero feminino para os resultados médios obtidos no WHOQOL-BREF.

\begin{tabular}{lccc}
\hline & Acondroplásicos & Não-acondroplásicos & $\mathbf{p}$ \\
\hline Físico & $72,0 \pm 8,5$ & $81,9 \pm 6,1$ & 0,002 \\
Psicológico & $73,7 \pm 14,1$ & $79,8 \pm 11,6$ & 0,241 \\
Social & $73,7 \pm 22,8$ & $78,2 \pm 16,2$ & 0,568 \\
Ambiental & $61,3 \pm 15,3$ & $70,4 \pm 12,7$ & 0,112 \\
Total & $69,6 \pm 11,3$ & $76,8 \pm 8,3$ & 0,077 \\
\hline
\end{tabular}

A QV reflete a percepção do indivíduo de sua posição na vida, no contexto da cultura, e do sistema de valores em que vive, e em relação aos seus objetivos, suas expectativas e seus padrões ${ }^{24}$. Tal percepção está diretamente influenciada pelo sexo, pela idade, pelo nível de escolaridade, pela condição socioeconômica e pela presença de incapacidades ${ }^{25}$.

Apajasalo et al. ${ }^{17}$ constataram que a QV de adultos portadores de displasias esqueléticas, em uma faixa etária dos 16 aos 54 anos, foi significativamente menor do que a do grupo- 
controle da mesma idade. A diferença entre esses pacientes resultou das queixas reportadas pelos adultos acondroplásicos nos parâmetros de mobilidade, atividades de lazer, atividade sexual e dores e desconfortos. No presente estudo, foi constatado que no domínio físico as mulheres acondroplásicas apresentaram resultados estatisticamente menores se comparados às mulheres do grupo-controle. Em concordância, Mahomed et al. ${ }^{16}$ constataram declínio no domínio físico associado ao aumento na freqüência de queixas musculoesqueléticas, estenoses espinhais e lombalgias crônicas.

A QV de adolescentes acondroplásicos na faixa etária entre 12 e 15 anos foi semelhante a dos adultos acondroplásicos. Os adolescentes apresentaram resultados menores nos âmbitos de mobilidade, escola, hobbies e amigos, atribuindo-se ao fato de que, no início da puberdade, há a tendência de as atividades sociais reduzirem-se e os amigos se afastarem ${ }^{22}$. Além disso, esses adolescentes se apresentaram significativamente menos satisfeitos com a aparência física em relação aos adolescentes do grupo-controle.

Mahomed et al. ${ }^{16}$ verificaram que para indivíduos acondroplásicos, depois da terceira década de vida, em ambos os sexos, apresentaram declínio estatisticamente significante no domínio físico se comparado à população em geral.

Embora no estudo de Apajasalo et al. ${ }^{17}$ o sexo não tenha mostrado associação estatisticamente significativa com a QV, os homens apresentaram a tendência a resultados maiores do que as mulheres. Tal tendência foi constatada de forma similar nas populações de adultos e adolescentes. Em contrapartida, no presente estudo foi observado que houve diferença estatisticamente significante no domínio psicológico entre os gêneros no grupo de acondroplásicos, o que sugere que as mulheres possuem mais problemas com auto-estima, imagem corporal, dificuldade de aceitação ou reportam tais problemas com maior facilidade que os homens; conforme

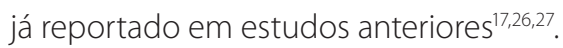

Em concordância neste estudo, ao compararmos indivíduos acondroplásicos e não-acondroplásicos do gênero masculino, não houve diferença estatisticamente significante em nenhum dos domínios da QV.

No presente estudo, não houve diferença estatisticamente significante no domínio ambiental, o qual se refere à segurança física e proteção, transportes, oportunidades de lazer, religião, entre outros; todavia, Shurka e Laron ${ }^{28}$ constataram problemas de mobilidade e de transporte público, reportados por adultos e adolescentes de baixa estatura.

De acordo com Tasker et al. ${ }^{29}$, a diminuição da caixa torácica; estenose do forame magno; hipertrofia das adenóides, a qual resulta obstrução das vias aéreas superiores; entre outros fatores, contribuem para a ocorrência de inúmeras complicações respiratórias, tais como apnéia do sono, insuficiência respiratória, asma brônquica, pneumonias recorrentes. As otites também são reportadas como complicações significativas da acondroplasia ${ }^{30}$.
Mahomed et al. ${ }^{16}$ constataram uma associação entre tais disfunções com o aspecto mental dos acondroplásicos, uma vez que processos alérgicos crônicos, otites, distúrbios do sono, doenças pulmonares crônicas, entre outros, resultaram diferenças estatisticamente significantes neste aspecto.

O domínio social da QV se refere à capacidade para o trabalho e à dependência de medicação ou de tratamentos do indivíduo. No presente estudo, tanto na comparação entre os grupos quanto na comparação entre os gêneros não houve diferença estatisticamente significante neste domínio. Em concordância, em um estudo realizado por Ho et al. ${ }^{31}$ ao se comparar a QV entre indivíduos acondroplásicos que sofreram cirurgia de descompressão cervicomedular e indivíduos acondroplásicos que não realizaram o procedimento, não houve diferença estatisticamente significante na percepção geral de saúde. Porém, é interessante ressaltar que daqueles que referiram ter um "excelente estado de saúde", 64\% pertenciam ao grupo que sofreu o procedimento cirúrgico de descompressão cervicomedular e 36\% pertenciam ao grupo que não realizou a cirurgia.

Por outro lado, um estudo com indivíduos acondroplásicos que relataram ter realizado algum procedimento cirúrgico (osteotomia, laminectomia lombar, adenoidectomia) mostrou que apresentaram resultados estatisticamente menores ao serem comparados com aqueles que não haviam sofrido qualquer intervenção cirúrgica. Entre alguns fatores para essa afirmação está o fato de essas cirurgias não terem sido realizadas precocemente, a fim de amenizar as complicações físicas e psicológicas já instaladas pela história da doença; e esses procedimentos não terem restaurado de forma efetiva a função física do paciente ${ }^{16}$.

Já em um estudo realizado por Gollust et al. ${ }^{21}$, a QV foi comparada entre indivíduos acondroplásicos e seus respectivos parentes de primeiro grau não-acondroplásicos. Foi constatado que os indivíduos com acondroplasia diferiram significativamente de seus parentes de primeiro grau ao se comparar auto-estima, educação, salário anual, percepção da condição e prática religiosa, em que os escores foram maiores para os indivíduos não-acondroplásicos.

A auto-estima, entre outros fatores, é uma das principais fontes para aceitação das incapacidades. A boa aceitação é indicativa de auto-estima maior, o que provavelmente refletirá nas habilidades individuais, facilitando a integração na sociedade ${ }^{32}$.

Além da auto-estima, a percepção quanto à seriedade da própria condição é uma característica importante associada à QV. Assim sendo, Gollust et al. ${ }^{21}$ relataram que os indivíduos não afetados que vêem a acondroplasia como uma condição "séria ou letal" apresentaram menores escores na QV em relação àqueles parentes não afetados que vêem a acondroplasia como uma condição "não séria". Membros de uma família que perceberam seu parente afetado como 
"um problema muito grave" apresentaram percepção negativa de sua própria QV.

Ainda, alguns autores têm referido a influência de outras disfunções sobre a QV. Bittencourt et al. ${ }^{33}$ estudaram a QV de deficientes visuais, cegos ou com baixa visão e constataram escores mais altos nos domínios psicológico e físico. Os domínios das relações sociais e do meio ambiente foram os mais prejudicados; uma vez que tais domínios referemse ao nível de independência, capacidade para o trabalho, segurança física e proteção, transportes, oportunidades de lazer etc. Já Castro et al. ${ }^{34}$ observaram a QV em pacientes dependentes do tabaco constatando que os domínios físico e ambiental foram os que apresentaram maior diferença. Porém, foi verificado prejuízo em todos os domínios avaliados pelo WHOQOL-BREF, destacando a influência da depressão, da ansiedade e dos sentimentos negativos sobre a QV.

São necessárias ações interdisciplinares que valorizem a independência e a autonomia dessas populações, buscando a redução das perdas funcionais, possibilitando o acesso a informações e objetivando a promoção da saúde ${ }^{33}$. A situação de doença ou deficiência é, para muitos, sinônimo de exclusão social, isolamento familiar e vulnerabilidade que geram estigmas individuais e coletivos. Incentivar novas pesquisas, despertar, conscientizar e fomentar discussões frente às diversidades contribui para a mudança cultural da sociedade e para a QV das pessoas portadoras de deficiências ${ }^{35}$.

\section{CONCLUSÃO}

Conclui-se que não houve diferença na QV ao se comparar indivíduos acondroplásicos e não-acondroplásicos; entretanto, nos domínios físico e psicológico, as mulheres acondroplásicas demonstraram menor satisfação com sua condição. É possível que o descontentamento com a imagem corporal e a aparência física seja explicado pela associação entre a visão destorcida delas frente à doença e pela submissão às exigências e às expectativas a que as mulheres em geral são compelidas.

\section{AGRADECIMENTOS}

Agradecemos à Associação Gente Pequena do Brasil pelo apoio para a realização do estudo e a todos os acondroplásicos que gentilmente participaram.

\section{REFERÊNCIAS}

1. Cotran RS, Kumar V, Collins T. Ossos, articulações e tumores de partes moles, In: Rosenberg A, (eds.) Robbins Pathologic Basis of Disease. Guanabara Koogan: Rio de Janeiro, 2000. p. 1092.
2. Francomano CA, Ortiz RI, Hefferson TW, Bellus GA, Turner E. Localization of the achondroplasia gene to the distal 2, 5 Mb of human chromosome 4p. Hum Mol Genet. 1994; 3:787-92.

3. Rousseau F, Bonaventure J, Legeai ML. Mutations in the gene encoding fibroblast growth factor receptor-3 in achondroplasia. Nature. 1994;371:252-4.

4. Griffiths AJF, Gelbart WM, Miller JH, Lewontin RC. A herança de genes, In Waters JC, Cole RW, Rieder CL, (eds.) Genética Moderna. Rio de Janeiro: Guanabara Koogan, 2001. p. 104-7.

5. Otto PG, Otto PA, Pessoa OF. Acondroplasia. In: Otto PG, Otto PA, Pessoa OF. Genética humana e clínica. São Paulo: Roca, 1998. p.153-8.

6. Shiang R, Thompson LM, Zhu V, Church DM, Fielder TJ, Bocian M, et al. Mutations in the transmembrane domain of FGFR3 cause the most common genetic form of dwarfism, achondroplasia. Cell. 1994;78:335-42.

7. Muenke M, Schell U. Fibroblast-growth-factor receptor mutations in human skeletal disorders. Trends in Genetics. 1995;11:308-13.

8. Kopits SE. Orthopedic complications of dwarfism. Clin Orthop Rel Res. 1976;114:153-79.

9. Marcondes EDV, Claudette HG. Acondroplasia: revisão com ênfase nos aspectos radiológicos. J Pediatr. 1987;9:103-13.

10. Hunter AGW, Bankier A, Rogers JG, Silence D, Scott CIJ. Medical complications of achondroplasia: a multicentre patient review. J Med Genet. 1998;35:705-712.

11. Fano V e Lejarraga H. Hallazgos frecuentes en la atención clínica de 96 ninõs con acondroplasia. Arch Argent. Pediatr. 2000;98(6):368.

12. Ain MC, Browne JA. Spinal arthrodesis with instrumentation for thoracolumbar kyphosis in pediatric achondroplasia. Spine. 2004;29:2075-80.

13. Hecht JT, Butler IJ. Neurologic morbidity associated with achondroplasia. J Child Neurol. 1990;5:84-97.

14. Reid CS, Pyeritz RE, Kopits SE, Maria BL, Wang H, McPherson RW, et al.. Cervicomedullary compression in young patients with achondroplasia: value of comprehensive neurologic and respiratory evaluation. J Pediatr. 1987;110(4):522-30.

15. Stokes DC, Phillips JA, Leonnard CO. Respiratory complications of achondroplasia. J Pediatr. 1983;102:534-41.

16. Mahomed N, Spellmann M, Goldberg JM. Functional health status of adults with achondroplasia. Am J Med Genet. 1998;78:30-5.

17. Apajasalo MSH, Rautonen J, Kaitila I. Health - related quality of life of patients with genetic skeletal dysplasias. Eur J Pediatr. 1998;157:114-21.

18. Folstein SE, Weiss J0, Mittelman F, Ross DJ. Impairment, psychiatric symptoms, and handicap in dwarfs. John Hopkins Med J. 1981;148:273-7.

19. Dein S. Does being religious help or hinder coping with chronic illness? A critical literature review. Palliat Med. 1997;11:291-8.

20. Koenig H. Religion, spirituality and medicine: Application to clinical practice. JAMA. 2000;284:1708

21. Gollust SE, Thompson RE, Gooding HC, Biesecker BB. Living with achondroplasia in an average-sized world: an assessment of quality of life. Am J Med Genet. 2003;120A :447-58.

22. Holmes CS, Karlsson JA, Thompson RG. Longitudinal evaluation behavior patterns in children with short stature, In: Stabler B, Underwood LE, (eds.) Slow grows the child: psychosocial aspects of growth delay. New Jersey: Lawrence Erlbaum Associates, 1986. p. 1-12.

23. Fleck MPA, Leal OF, Louzada S, Xavier M, Chachamovit E, Vieira G et al. Development of the Portuguese version of the OMS evaluation instrument of quality of life. Rev Bras Psiquiatr. 1999;21:1.

24. OMS: Organización Mundial de la Salud. Promoción de la salud; glosario. Genebra, 1998.

25. Lebrão ML, Laurenti R. Condições de saúde. In: Lebrão ML, Duarte YAO. SABE: saúde, bemestar e envelhecimento: 0 Projeto SABE no município de São Paulo: uma abordagem inicial. Organização Pan-Americana da Saúde. Brasília, 2003. p. 73-91.

26. Sweeting H. Reversals of fortune? Sex differences in health in childhood and adolescence. Soc Sci Med. 1995;40:77-90. 
27. Tiblin G, Bengtsson C, Furunes B, Lapidus L. Prevalence of symptoms in men and women. The population studies of men and women in Gothenburg, Sweden. Scand J Prim Health Care. 1990;8:9-17.

28. Shurka E, Laron Z. Adjustment and rehabilitation problems of children and adolescents with growth retardation: familiar dwarfism with high plasma immunoreactive human growth hormone. Israel J Med Sci. 1975;11:352-7.

29. Tasker RC, Dundas I, Laverty A, Flecther M, Lane R, Stocks J. Distinct patterns of respiratory difficulty in young children with achondroplasia: a clinical, sleep and lung function study. Arch Dis Child. 1998:79:99-108.

30. Cohen MM. Dysmorphic syndromes with craniofacial manifestations. In: Stewart RE, Prescott G. (eds.). Oral facial genetics. St Louis: Mosby, 1976. p 523-5.
31. Ho NC, Guarnieri M, Brant LJ, Park SS, Sun B, North M et al. Living with achondroplasia: quality of life evaluation following cervico-medullary descompression. Am J Med Genet. 2004;131A:163-7.

32. Li L, Moore D. Acceptance of disability and its correlates. J Soc Psychol. 1998;138:13-25.

33. Bittencourt ZZLC, Hoehne EL. Quality of life of visually impaired people. Med Ribeirão Preto. 2006;39(2):260-4.

34. Castro MG, Oliveira MS, Moraes JFD, Miguel AC, Araújo RB. Quality of life and severity of tobacco dependence. Rev. Psiq. Clin. 2007;34(2):61-7.

35. Néri M. Retratos das deficiências no Brasil (PPD). Rio de Janeiro: FGV/IBRE, CPS, 2003. p. 250 